June 25, 2019

\title{
Systematic Application of the Principle of Detailed Balancing to Complex Homogeneous Chemical Reaction Mechanisms
}

\author{
D. M. Stanbury* \& D. Hoffman ${ }^{ \pm}$ \\ *Dept. of Chemistry and Biochemistry, ${ }^{ \pm}$Dept. of Mathematics and Statistics \\ Auburn University, Auburn, AL 36849, USA
}

\section{Supporting Information}

Systematic method to determine whether a mechanism contains any illegal loops.

Row reduction of the full stoichiometric matrix augmented with the identity matrix reveals a basis set of loops. If one of these loops contains coefficients that all have the same sign for the irreversible steps then it is illegal. However, sometimes there will be illegal loops that exist only as linear combinations of the loops within linked sets of loops found by the row reduction mentioned above. Here we present a systematic way to determine whether the mechanism includes any illegal loops, and we illustrate the method with an example of a legal mechanism and an illegal mechanism.

The first step is to extract the submatrix that contains the basis set of loops described above. Identify any linked sets of loops, and for each linked set perform the following operations. Second, remove the columns corresponding to reversible steps and add an identity matrix to the right. Third, perform a row reduction on this matrix. Add labels so the coefficients of the linear combinations can be identified. Examine the result to see whether there are any rows not containing matrix elements of opposite sign. Such rows correspond to illegal loops. If no such rows are found there may still be illegal loops existing as linear combinations. Step 4: Remove any rows consisting entirely of zeros and then transpose the matrix. Step 5: add a row of -1 at the bottom of the matrix to create matrix $\mathrm{m}$. Step 6: create a vector $\mathrm{c}$ that consists of as many -1 's as the number of columns in matrix $\mathrm{m}$. Step 7: create a vector $b$ that has as many elements as the number of rows in matrix $m$ and consists of 0 's except for the last entry, which should be -120 . Step 8: perform the Mathematica operation LinearProgramming[c,m,b]. Step 9: compute the dot product of the output of the LinearProgramming and vector c. If the result is -120 then there is at least one illegal loop. Otherwise the mechanism contains no illegal loops.

The logic behind this method is described here. The Mathematica operation LinearProgramming[c,m.b] outputs a vector $\mathrm{x}$ minimizing c.x, subject to certain conditions. Without the row of -1 's added in step 5 , if there was a solution, there would be solutions $\mathrm{x}$ with 
c.x a negative number of arbitrarily large absolute value, i.e. no minimum. The added row, and the -120 in step 7 , ensures that c.x is at least -120 . The value -120 was chosen in an effort to avoid fractions. If the software outputs an $\mathrm{x}$ with fractions, they may be cleared by multiplying through by a common denominator.

\section{Example of a legal mechanism}

This procedure is illustrated with the 19-step mechanism proposed for the reaction of bromate with MESNA. ${ }^{1}$ The full stoichiometric matrix augmented with the identity matrix is shown below:

\begin{tabular}{|c|c|c|c|c|c|c|c|c|c|c|c|c|c|c|c|c|c|c|c|c|c|c|c|c|c|c|c|c|c|c|c|}
\hline 0 & Br03- & RSH & $\mathrm{H}+$ & $\mathrm{HBrO2}$ & $\mathrm{RSOH}$ & $\mathrm{HOBr}$ & $\mathrm{Br}-$ & $\mathrm{Br} 2$ & $\mathrm{H} 2 \mathrm{O}$ & $\mathrm{RSO} 2 \mathrm{H}$ & $\mathrm{RSO} 3 \mathrm{H}$ & RSSR & $\mathrm{BrO2}$ & RS. & 0 & 0 & 0 & 0 & 0 & 0 & 0 & 0 & 0 & 0 & 0 & 0 & 0 & 0 & 0 & 0 & 0 \\
\hline M1 & -1 & -1 & -1 & 1 & 1 & 0 & 0 & 0 & 0 & 0 & 0 & 0 & $\odot$ & 0 & 1 & 0 & 0 & 0 & 0 & $0 \quad 0$ & 0 & 0 & 0 & 0 & 0 & 0 & 0 & 0 & $\odot$ & 0 & 0 \\
\hline M2 & 0 & -1 & 0 & -1 & 1 & 1 & 0 & $\odot$ & $\odot$ & 0 & $\odot$ & $\odot$ & 0 & 0 & 0 & 1 & $\odot$ & 0 & 0 & $\odot \quad 0$ & $\odot$ & 0 & 0 & 0 & 0 & 0 & 0 & $\odot$ & 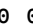 & 0 & 0 \\
\hline M3 & 0 & -1 & 1 & 0 & 1 & -1 & 1 & 0 & 0 & 0 & 0 & $\odot$ & 0 & 0 & 0 & 0 & 1 & 0 & 0 & 0 & 0 & 0 & 0 & 0 & 0 & 0 & 0 & 0 & $\odot$ & 0 & 0 \\
\hline M4R & -1 & 0 & -2 & 1 & 0 & 1 & -1 & 0 & 0 & 0 & 0 & 0 & 0 & 0 & 0 & 0 & 0 & 1 & 0 & 0 & 0 & 0 & 0 & 0 & 0 & 0 & 0 & 0 & $\odot$ & 0 & 0 \\
\hline M5R & 0 & 0 & -1 & -1 & 0 & 2 & -1 & 0 & 0 & 0 & 0 & 0 & 0 & 0 & 0 & 0 & 0 & 0 & 1 & 0 & 0 & 0 & 0 & 0 & 0 & 0 & 0 & $\odot$ & $\odot$ & 0 & 0 \\
\hline M6R & 0 & 0 & -1 & 0 & 0 & -1 & -1 & 1 & 1 & 0 & 0 & 0 & 0 & 0 & 0 & 0 & 0 & 0 & 0. & 1 & $\odot$ & 0 & 0 & 0 & 0 & 0 & 0 & 0 & 0 & 0 & 0 \\
\hline M7 & 0 & -1 & 1 & 0 & 1 & -1 & 1 & 0 & 0 & 0 & 0 & 0 & 0 & $\odot$ & 0 & 0 & 0 & 0 & 0 & 0 & 0 & 0 & 0 & 0 & 0 & 0 & 0 & $\odot$ & $\odot$ & 0 & 0 \\
\hline M8 & 0 & 0 & 1 & 0 & -1 & -1 & 1 & 0 & 0 & 1 & 0 & 0 & 0 & 0 & 0 & 0 & 0 & $\odot$ & 0 & 0 & 1 & 0 & 0 & 0 & 0 & 0 & 0 & 0 & $\odot$ & 0 & 0 \\
\hline M9 & 0 & 0 & 1 & 0 & 0 & -1 & 1 & 0 & 0 & -1 & 1 & 0 & 0 & 0 & 0 & 0 & 0 & $\odot$ & 0 & $\odot$ & $\odot$ & 1 & 0 & 0 & 0 & $\odot$ & $\odot$ & $\odot$ & $\odot$ & 0 & 0 \\
\hline M10 & $\odot$ & -1 & 2 & $\odot$ & 1 & $\odot$ & 2 & -1 & -1 & 0 & $\odot$ & 0 & 0 & $\odot$ & 0 & 0 & 0 & $\odot$ & 0 & $\odot$ & $\odot$ & 0 & 1 & 0 & 0 & $\odot$ & 0 & $\odot$ & $\odot$ & 0 & 0 \\
\hline M11 & 0 & 0 & 2 & 0 & -1 & 0 & 2 & -1 & -1 & 1 & 0 & 0 & 0 & 0 & 0 & 0 & 0 & 0 & 0 & 0 & $\odot$ & 0 & 0 & 1 & 0 & 0 & 0 & 0 & 0 & 0 & 0 \\
\hline M12 & $\odot$ & $\odot$ & 2 & 0 & $\odot$ & 0 & 2 & -1 & -1 & -1 & 1 & 0 & 0 & $\odot$ & 0 & 0 & 0 & $\odot$ & 0 & $\odot$ & $\odot$ & 0 & $\odot$ & $\odot$ & 1 & $\odot$ & $\odot$ & $\odot$ & $\odot$ & 0 & 0 \\
\hline M13R & 0 & 0 & $\odot$ & 0 & 1 & 0 & 0 & $\odot$ & $\odot$ & -2 & 1 & 0 & 0 & 0 & 0 & 0 & 0 & $\odot$ & 0 & $\odot$ & $\odot$ & 0 & 0 & 0 & 0 & 1 & 0 & $\odot$ & $\odot$ & $\odot$ & 0 \\
\hline M14R & 0 & -1 & 0 & 0 & -1 & 0 & 0 & 0 & 1 & 0 & 0 & 1 & 0 & 0 & 0 & 0 & 0 & 0 & 0 & 0 & 0 & 0 & 0 & 0 & 0 & 0 & 1 & 0 & $\odot$ & 0 & 0 \\
\hline M15 & $\odot$ & 0 & 1 & $\odot$ & 2 & -1 & 1 & 0 & -1 & 0 & 0 & -1 & 0 & $\odot$ & 0 & 0 & 0 & 0 & 0 & $\odot$ & $\odot$ & 0 & 0 & 0 & 0 & $\odot$ & 0 & 1 & $\odot$ & 0 & 0 \\
\hline M16 & 0 & 0 & 2 & 0 & 2 & 0 & 2 & -1 & -2 & $\odot$ & 0 & -1 & 0 & 0 & 0 & 0 & 0 & $\odot$ & 0 & $\odot$ & 0 & 0 & $\odot$ & 0 & 0 & $\odot$ & 0 & $\odot$ & 1 & $\odot$ & 0 \\
\hline M17R & -1 & $\odot$ & -1 & -1 & 0 & 0 & 0 & $\odot$ & 1 & $\odot$ & $\odot$ & 0 & 2 & $\odot$ & $\odot$ & 0 & $\odot$ & 0 & 0 & $\odot$ & 0 & 0 & $\odot$ & 0 & 0 & $\odot$ & $\odot$ & 0 & $\odot$ & $\odot$ & 0 \\
\hline M18R & 0 & -1 & 0 & 1 & 0 & 0 & 0 & 0 & 0 & $\odot$ & 0 & $\odot$ & -1 & 1 & 0 & 0 & $\odot$ & $\odot$ & 0 & 0 & 0 & 0 & $\odot$ & 0 & 0 & 0 & $\odot$ & $\odot$ & $\odot$ & 1 & 0 \\
\hline M19 & 0 & 0 & 0 & 0 & 0 & 0 & 0 & 0 & 0 & 0 & 0 & 1 & $\odot$ & -2 & $\odot$ & 0 & 0 & 0 & 0 & 0 & 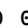 & $\odot$ & 0 & 0 & 0 & 0 & 0 & & $\odot$ & $\odot$ & 1 \\
\hline
\end{tabular}

Row reduction generates the basis set of 10 loops shown below:

\begin{tabular}{|c|c|c|c|c|c|c|c|c|c|c|c|c|c|c|c|c|c|c|c|}
\hline 1 & 0 & 0 & 0 & 0 & 0 & 0 & 0 & 0 & 0 & 0 & 0 & 0 & 1 & 0 & 0 & -1 & -2 & -1 & L1 \\
\hline 0 & 1 & 0 & 0 & -1 & 0 & 0 & 0 & 0 & 0 & 0 & 0 & 0 & -1 & -1 & 0 & 0 & 0 & 0 & L2 \\
\hline 0 & $\odot$ & 1 & 0 & 0 & 0 & $\odot$ & 0 & $\odot$ & 0 & 0 & 0 & 0 & -1 & -1 & 0 & $\odot$ & 0 & 0 & L3 \\
\hline 0 & $\odot$ & $\odot$ & 1 & 0 & 0 & $\odot$ & $\odot$ & 0 & 0 & 0 & 0 & 0 & 2 & 1 & 0 & -1 & -2 & -1 & L4 \\
\hline$\odot$ & $\odot$ & 0 & $\odot$ & $\odot$ & 1 & $\odot$ & $\odot$ & 0 & 0 & 0 & 0 & 0 & 0 & -1 & 1 & 0 & 0 & 0 & L5 \\
\hline 0 & 0 & 0 & 0 & 0 & 0 & 1 & 0 & 0 & 0 & 0 & 0 & 0 & -1 & -1 & $\odot$ & $\odot$ & $\odot$ & $\odot$ & L6 \\
\hline 0 & 0 & 0 & 0 & 0 & 0 & 0 & 1 & 0 & 0 & 0 & -1 & 1 & 0 & -1 & 1 & 0 & 0 & 0 & L7 \\
\hline$\odot$ & $\odot$ & 0 & $\odot$ & 0 & 0 & 0 & $\odot$ & 1 & 0 & 0 & -1 & 0 & 0 & -1 & 1 & 0 & 0 & 0 & L8 \\
\hline$\odot$ & $\odot$ & 0 & $\odot$ & $\odot$ & $\odot$ & $\odot$ & 0 & $\odot$ & 1 & 0 & 0 & 0 & -1 & $\odot$ & -1 & 0 & 0 & 0 & L9 \\
\hline 0 & 0 & 0 & 0 & 0 & 0 & 0 & 0 & 0 & 0 & 1 & -1 & 1 & 0 & $\Theta$ & 0 & $\Theta$ & $\Theta$ & $\Theta$ & L10 \\
\hline & M2 & M3 & M4R & M5R & $\mathrm{M} 6 \mathrm{R}$ & M7 & M8 & M9 & M10 & M11 & M12 & M13R & M14R & M15 & M16 & M17R & M18R & M19 & $\square$ \\
\hline
\end{tabular}

All 10 of these loops are legal and linked and thus should be examined together for linear combinations that are illegal. An identity matrix is added to the right. Columns corresponding to reversible reactions are labeled with the suffix R. They are removed. Column and row labels are removed and row reduction is performed again: 


$$
\text { RowReduce }\left[\begin{array}{ccccccccccccccccccccccc}
1 & 0 & 0 & 0 & 0 & 0 & 0 & 0 & 0 & 0 & 0 & -1 & 1 & 0 & 0 & 0 & 0 & 0 & 0 & 0 & 0 & 0 \\
0 & 1 & 0 & 0 & 0 & 0 & 0 & 0 & 0 & -1 & 0 & 0 & 0 & 1 & 0 & 0 & 0 & 0 & 0 & 0 & 0 & 0 \\
0 & 0 & 1 & 0 & 0 & 0 & 0 & 0 & 0 & -1 & 0 & 0 & 0 & 0 & 1 & 0 & 0 & 0 & 0 & 0 & 0 & 0 \\
0 & 0 & 0 & 0 & 0 & 0 & 0 & 0 & 0 & 1 & 0 & -1 & 0 & 0 & 0 & 1 & 0 & 0 & 0 & 0 & 0 & 0 \\
0 & 0 & 0 & 0 & 0 & 0 & 0 & 0 & 0 & -1 & 1 & 0 & 0 & 0 & 0 & 0 & 1 & 0 & 0 & 0 & 0 & 0 \\
0 & 0 & 0 & 1 & 0 & 0 & 0 & 0 & 0 & -1 & 0 & 0 & 0 & 0 & 0 & 0 & 0 & 1 & 0 & 0 & 0 & 0 \\
0 & 0 & 0 & 0 & 1 & 0 & 0 & 0 & -1 & -1 & 1 & 0 & 0 & 0 & 0 & 0 & 0 & 0 & 1 & 0 & 0 & 0 \\
0 & 0 & 0 & 0 & 0 & 1 & 0 & 0 & -1 & -1 & 1 & 0 & 0 & 0 & 0 & 0 & 0 & 0 & 0 & 1 & 0 & 0 \\
0 & 0 & 0 & 0 & 0 & 0 & 1 & 0 & 0 & 0 & -1 & 0 & 0 & 0 & 0 & 0 & 0 & 0 & 0 & 0 & 1 & 0 \\
0 & 0 & 0 & 0 & 0 & 0 & 0 & 1 & -1 & 0 & 0 & 0 & 0 & 0 & 0 & 0 & 0 & 0 & 0 & 0 & 0 & 1
\end{array}\right) \text { // MatrixForm }
$$

This generates the following matrix after labels are added:

\begin{tabular}{|c|c|c|c|c|c|c|c|c|c|c|c|c|c|c|c|c|c|c|c|c|c|c|}
\hline 1 & 0 & 0 & 0 & 0 & 0 & 0 & 0 & 0 & 0 & 0 & -1 & 1 & 0 & 0 & 0 & 0 & 0 & 0 & 0 & 0 & $\odot$ & L1 ' \\
\hline 0 & 1 & 0 & 0 & 0 & 0 & 0 & 0 & 0 & 0 & 0 & -1 & 0 & 1 & 0 & 1 & $\odot$ & 0 & 0 & 0 & 0 & 0 & L2 ' \\
\hline 0 & 0 & 1 & 0 & $\odot$ & 0 & 0 & 0 & 0 & 0 & 0 & -1 & 0 & 0 & 1 & 1 & 0 & 0 & 0 & 0 & $\odot$ & 0 & L3 ' \\
\hline$\odot$ & $\odot$ & $\odot$ & 1 & $\odot$ & 0 & $\odot$ & $\odot$ & $\odot$ & $\odot$ & 0 & -1 & 0 & 0 & 0 & 1 & 0 & 1 & 0 & 0 & 0 & $\odot$ & L4 ' \\
\hline$\odot$ & $\odot$ & $\odot$ & $\odot$ & 1 & 0 & $\odot$ & 0 & -1 & $\odot$ & 0 & $\odot$ & $\odot$ & 0 & 0 & 0 & -1 & 0 & 1 & $\odot$ & 0 & 0 & L5 ' \\
\hline 0 & $\odot$ & 0 & 0 & 0 & 1 & $\odot$ & 0 & -1 & 0 & 0 & 0 & $\odot$ & 0 & 0 & 0 & -1 & 0 & 0 & 1 & 0 & 0 & L6 ' \\
\hline 0 & 0 & $\odot$ & 0 & 0 & 0 & 1 & 0 & 0 & $\odot$ & 0 & -1 & 0 & 0 & 0 & 1 & 1 & 0 & $\odot$ & 0 & 1 & 0 & L7 ' \\
\hline 0 & 0 & $\odot$ & 0 & 0 & $\odot$ & $\odot$ & 1 & -1 & 0 & 0 & 0 & 0 & 0 & 0 & 0 & 0 & 0 & 0 & 0 & 0 & 1 & L8 ' \\
\hline 0 & 0 & 0 & 0 & $\odot$ & 0 & 0 & 0 & 0 & 1 & 0 & -1 & 0 & 0 & 0 & 1 & 0 & 0 & 0 & 0 & 0 & 0 & L9' \\
\hline$\odot$ & $\odot$ & 0 & $\odot$ & $\odot$ & $\odot$ & $\odot$ & $\odot$ & 0 & $\odot$ & 1 & -1 & $\odot$ & $\odot$ & $\odot$ & 1 & 1 & $\odot$ & 0 & $\odot$ & 0 & $\odot$ & L10 ' \\
\hline M1 & M2 & M3 & M7 & M8 & M9 & M10 & M11 & M12 & M15 & M16 & M19 & L1 & L2 & L3 & L4 & L5 & L6 & L7 & L8 & L9 & L10 & $\square$ \\
\hline
\end{tabular}

The right hand submatrix and labels are removed and the matrix is transposed as in

$\operatorname{Transpose}\left[\left(\begin{array}{cccccccccccc}1 & 0 & 0 & 0 & 0 & 0 & 0 & 0 & 0 & 0 & 0 & -1 \\ 0 & 1 & 0 & 0 & 0 & 0 & 0 & 0 & 0 & 0 & 0 & -1 \\ 0 & 0 & 1 & 0 & 0 & 0 & 0 & 0 & 0 & 0 & 0 & -1 \\ 0 & 0 & 0 & 1 & 0 & 0 & 0 & 0 & 0 & 0 & 0 & -1 \\ 0 & 0 & 0 & 0 & 1 & 0 & 0 & 0 & -1 & 0 & 0 & 0 \\ 0 & 0 & 0 & 0 & 0 & 1 & 0 & 0 & -1 & 0 & 0 & 0 \\ 0 & 0 & 0 & 0 & 0 & 0 & 1 & 0 & 0 & 0 & 0 & -1 \\ 0 & 0 & 0 & 0 & 0 & 0 & 0 & 1 & -1 & 0 & 0 & 0 \\ 0 & 0 & 0 & 0 & 0 & 0 & 0 & 0 & 0 & 1 & 0 & -1 \\ 0 & 0 & 0 & 0 & 0 & 0 & 0 & 0 & 0 & 0 & 1 & -1\end{array}\right)\right] / /$ MatrixForm

A row of -1 's is added to the bottom of the transposed matrix to create matrix m: 


$$
\left(\begin{array}{cccccccccc}
1 & 0 & 0 & 0 & 0 & 0 & 0 & 0 & 0 & 0 \\
0 & 1 & 0 & 0 & 0 & 0 & 0 & 0 & 0 & 0 \\
0 & 0 & 1 & 0 & 0 & 0 & 0 & 0 & 0 & 0 \\
0 & 0 & 0 & 1 & 0 & 0 & 0 & 0 & 0 & 0 \\
0 & 0 & 0 & 0 & 1 & 0 & 0 & 0 & 0 & 0 \\
0 & 0 & 0 & 0 & 0 & 1 & 0 & 0 & 0 & 0 \\
0 & 0 & 0 & 0 & 0 & 0 & 1 & 0 & 0 & 0 \\
0 & 0 & 0 & 0 & 0 & 0 & 0 & 1 & 0 & 0 \\
0 & 0 & 0 & 0 & -1 & -1 & 0 & -1 & 0 & 0 \\
0 & 0 & 0 & 0 & 0 & 0 & 0 & 0 & 1 & 0 \\
0 & 0 & 0 & 0 & 0 & 0 & 0 & 0 & 0 & 1 \\
-1 & -1 & -1 & -1 & 0 & 0 & -1 & 0 & -1 & -1 \\
-1 & -1 & -1 & -1 & -1 & -1 & -1 & -1 & -1 & -1
\end{array}\right)
$$

Vectors $\mathrm{c}$ and $\mathrm{b}$ are created and combined with matrix $\mathrm{m}$ for linear programming:

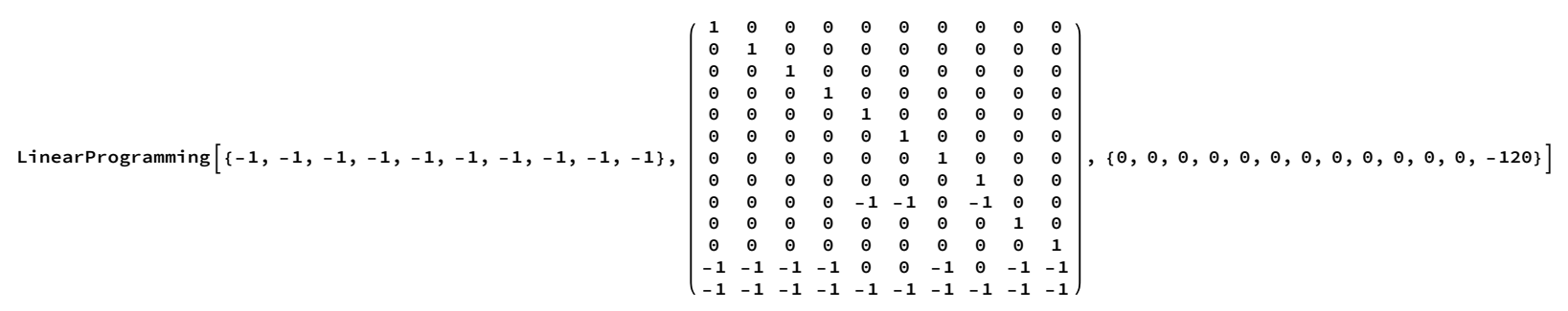

The result is

$\{\odot, \odot, \odot, \odot, \odot, \odot, \odot, \odot, \odot, \odot\}$

The dot product of this result and vector $\mathrm{c}$ is

$\{\odot, 0, \odot, \odot, \odot, \odot, \odot, 0,0, \odot\} \cdot\{-1,-1,-1,-1,-1,-1,-1,-1,-1,-1\}$

The final result is zero, which proves that there are no illegal loops in the proposed mechanism.

\section{Example of an illegal mechanism identified in the first row reduction}

The mechanism proposed for the copper-catalyzed oxidation of $\mathrm{S}_{2} \mathrm{O}_{3}{ }^{2-}$ by $\mathrm{S}_{2} \mathrm{O}_{8}{ }^{2-}$ provides a simple example where an illegal loop is identified in the first row reduction. ${ }^{2}$ The stoichiometric matrix is shown below: 


$\left(\begin{array}{cccccccccccc}0 & \mathrm{Cu} 2+ & \mathrm{S} 2032- & \mathrm{Cu}+ & \mathrm{S} 203-\mathrm{S} 2082- & \mathrm{S} 042- & \mathrm{S} 04- & \mathrm{S} 203 & \mathrm{~S} 4062- & \mathrm{Cu}+ & \mathrm{S} 04 \\ \mathrm{~B} 1 & -1 & -1 & 1 & 1 & 0 & 0 & 0 & 0 & 0 & 0 & 0 \\ \mathrm{~B} 2 & 0 & 0 & 0 & -1 & -1 & 1 & 1 & 1 & 0 & 0 & 0 \\ \text { B3 } & 0 & -1 & 0 & 2 & 0 & 0 & 0 & -1 & 0 & 0 & 0 \\ \text { B4 } & 0 & 0 & 0 & -2 & 0 & 0 & 0 & 0 & 1 & 0 & 0 \\ \text { B5 } & 1 & 0 & -1 & 0 & -1 & 1 & 1 & 0 & 0 & 0 & 0 \\ \text { B6 } & -1 & 0 & 0 & 0 & -1 & 1 & 1 & 0 & 0 & 1 & 0 \\ \text { B7 } & 1 & 0 & 0 & 0 & 0 & 0 & -1 & 0 & 0 & -1 & 1 \\ \text { B8 } & 0 & 0 & 0 & 0 & 0 & -1 & 2 & 0 & 0 & 0 & -1 \\ \text { B9 } & 0 & 0 & 0 & 0 & 1 & 0 & -2 & 0 & 0 & 0 & 0\end{array}\right)$

An identity matrix is added and the row reduction operation is specified:

RowReduce $\left[\left(\begin{array}{cccccccccccccccccccc}-1 & -1 & 1 & 1 & 0 & 0 & 0 & 0 & 0 & 0 & 0 & 1 & 0 & 0 & 0 & 0 & 0 & 0 & 0 & 0 \\ 0 & 0 & 0 & -1 & -1 & 1 & 1 & 1 & 0 & 0 & 0 & 0 & 1 & 0 & 0 & 0 & 0 & 0 & 0 & 0 \\ 0 & -1 & 0 & 2 & 0 & 0 & 0 & -1 & 0 & 0 & 0 & 0 & 0 & 1 & 0 & 0 & 0 & 0 & 0 & 0 \\ 0 & 0 & 0 & -2 & 0 & 0 & 0 & 0 & 1 & 0 & 0 & 0 & 0 & 0 & 1 & 0 & 0 & 0 & 0 & 0 \\ 1 & 0 & -1 & 0 & -1 & 1 & 1 & 0 & 0 & 0 & 0 & 0 & 0 & 0 & 0 & 1 & 0 & 0 & 0 & 0 \\ -1 & 0 & 0 & 0 & -1 & 1 & 1 & 0 & 0 & 1 & 0 & 0 & 0 & 0 & 0 & 0 & 1 & 0 & 0 & 0 \\ 1 & 0 & 0 & 0 & 0 & 0 & -1 & 0 & 0 & -1 & 1 & 0 & 0 & 0 & 0 & 0 & 0 & 1 & 0 & 0 \\ 0 & 0 & 0 & 0 & 0 & -1 & 2 & 0 & 0 & 0 & -1 & 0 & 0 & 0 & 0 & 0 & 0 & 0 & 1 & 0 \\ 0 & 0 & 0 & 0 & 1 & 0 & -2 & 0 & 0 & 0 & 0 & 0 & 0 & 0 & 0 & 0 & 0 & 0 & 0 & 1\end{array}\right)\right]$ // MatrixForm

The result is

$\left(\begin{array}{ccccccccccccccccccccc}1 & 0 & 0 & 0 & 0 & 0 & 0 & 1 & -\frac{1}{2} & -1 & 0 & 0 & 1 & 0 & -\frac{1}{2} & 0 & 0 & 1 & 1 & 1 & \square \\ 0 & 1 & 0 & 0 & 0 & 0 & 0 & 1 & -1 & 0 & 0 & 0 & 0 & -1 & -1 & 0 & 0 & 0 & 0 & 0 & \square \\ 0 & 0 & 1 & 0 & 0 & 0 & 0 & 2 & -1 & -1 & 0 & 0 & 2 & 0 & -1 & -1 & 0 & 1 & 1 & 1 & \square \\ 0 & 0 & 0 & 1 & 0 & 0 & 0 & 0 & -\frac{1}{2} & 0 & 0 & 0 & 0 & 0 & -\frac{1}{2} & 0 & 0 & 0 & 0 & 0 & \square \\ 0 & 0 & 0 & 0 & 1 & 0 & 0 & 2 & -1 & 0 & -2 & 0 & 2 & 0 & -1 & 0 & 0 & 0 & 2 & 3 & \square \\ 0 & 0 & 0 & 0 & 0 & 1 & 0 & 2 & -1 & 0 & -1 & 0 & 2 & 0 & -1 & 0 & 0 & 0 & 1 & 2 & \square \\ 0 & 0 & 0 & 0 & 0 & 0 & 1 & 1 & -\frac{1}{2} & 0 & -1 & 0 & 1 & 0 & -\frac{1}{2} & 0 & 0 & 0 & 1 & 1 & \square \\ 0 & 0 & 0 & 0 & 0 & 0 & 0 & 0 & 0 & 0 & 0 & 1 & -1 & -1 & 0 & 1 & 0 & 0 & 0 & 0 & \mathrm{~L} 1 \\ 0 & 0 & 0 & 0 & 0 & 0 & 0 & 0 & 0 & 0 & 0 & 0 & 0 & 0 & 0 & 0 & 1 & 1 & 1 & 1 & \mathrm{~L} 2 \\ \square & \square & \square & \square & \square & \square & \square & \square & \square & \square & \square & \mathrm{B} 1 & \mathrm{~B} 2 & \mathrm{~B} 3 & \mathrm{~B} 4 & \mathrm{~B} 5 & \mathrm{~B} 6 & \mathrm{~B} 7 & \mathrm{~B} 8 & \mathrm{~B} 9 \mathrm{R} & \square\end{array}\right)$

L2 is identified as being illegal, because the nonzero coefficients on the right-hand side are all the same sign. 


\section{Example of an illegal mechanism identified in the second row reduction}

An example where illegal loops are not identified until the second row reduction is provided by the mechanism proposed for the reaction of periodate with thiosulfate. ${ }^{3}$ The complete stoichiometric matrix plus identity matrix is given below:

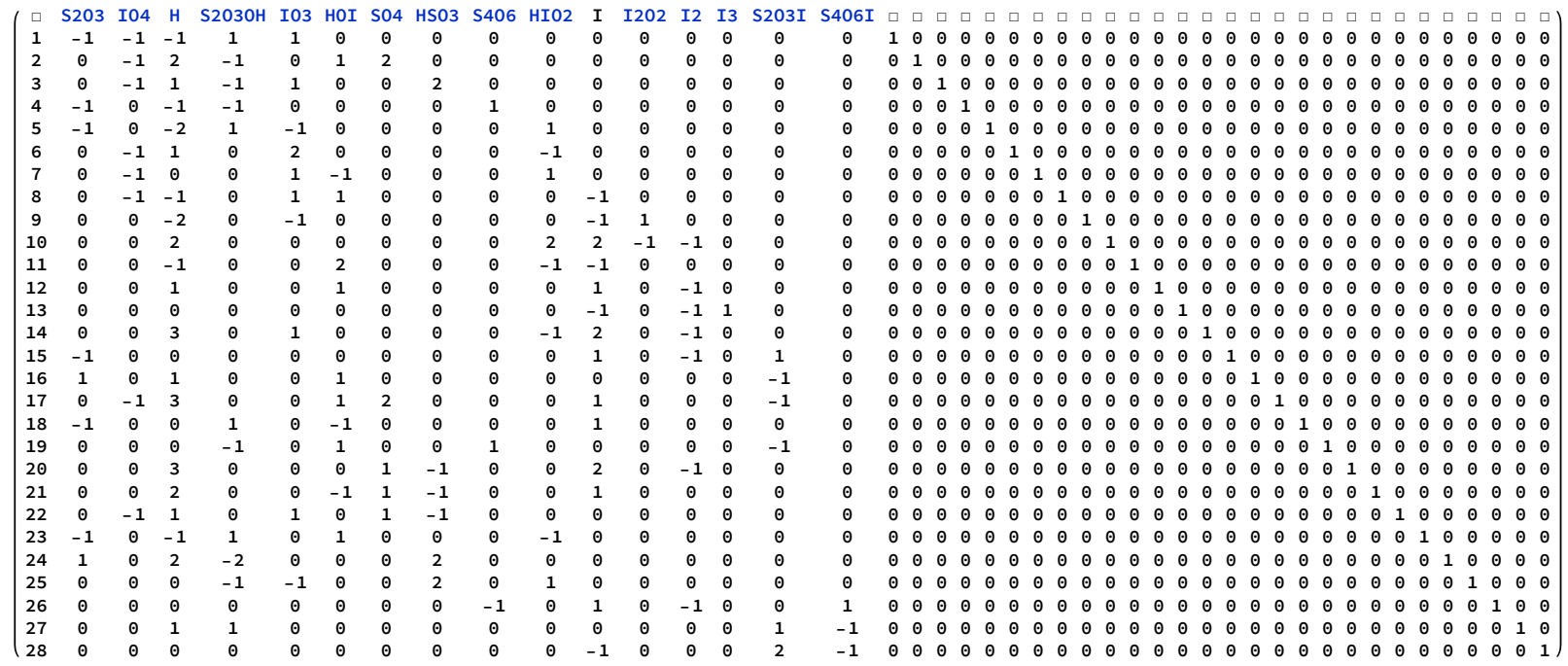

Row reduction reveals a basis set of 16 loops:

\begin{tabular}{|c|c|c|c|c|c|c|c|c|c|c|c|c|c|c|c|c|c|c|c|c|c|c|c|c|c|c|c|c|}
\hline 1 & 0 & $\odot$ & $\odot$ & $\odot$ & $\odot$ & $\odot$ & $\odot$ & 0 & 0 & $\odot$ & 0 & $\odot$ & $\odot$ & $\odot$ & $\odot$ & 0 & -1 & $\odot$ & $\odot$ & 1 & -1 & $\odot$ & $\odot$ & $\odot$ & $\odot$ & 0 & 0 & L1 \\
\hline 0 & 1 & 0 & 0 & 0 & 0 & $\odot$ & 0 & $\odot$ & 0 & 0 & $\odot$ & $\odot$ & 0 & 0 & $\odot$ & $\odot$ & 1 & 0 & 0 & -1 & -1 & -1 & 0 & -1 & $\odot$ & $\odot$ & $\odot$ & L2 \\
\hline 0 & 0 & 1 & 0 & 0 & 0 & $\odot$ & 0 & $\odot$ & 0 & 0 & 0 & $\odot$ & 0 & 0 & 0 & 0 & -1 & 0 & 0 & 1 & -1 & 0 & -1 & 0 & $\odot$ & $\odot$ & $\odot$ & L3 \\
\hline 0 & 0 & 0 & 1 & 0 & 0 & 0 & 0 & $\odot$ & 0 & 0 & 0 & 0 & 0 & 0 & 0 & 0 & -1 & $\odot$ & -1 & 1 & 0 & 0 & 0 & 0 & 1 & 2 & -1 & L4 \\
\hline 0 & 0 & 0 & 0 & 1 & $\odot$ & 0 & $\odot$ & $\odot$ & 0 & 0 & 0 & $\odot$ & $\odot$ & $\odot$ & 0 & 0 & 0 & 0 & 0 & 0 & 0 & 0 & 1 & -1 & 0 & 0 & 0 & L5 \\
\hline$\odot$ & 0 & 0 & 0 & $\odot$ & 1 & $\odot$ & 0 & $\odot$ & $\odot$ & 0 & $\odot$ & $\odot$ & 0 & 0 & 0 & $\odot$ & -1 & $\odot$ & 0 & 1 & -1 & 0 & -1 & 1 & $\odot$ & $\odot$ & $\odot$ & L6 \\
\hline$\odot$ & $\odot$ & 0 & 0 & $\odot$ & 0 & 1 & 0 & $\odot$ & $\odot$ & 0 & $\odot$ & $\odot$ & 0 & 0 & $\odot$ & $\odot$ & -1 & $\odot$ & $\odot$ & 1 & -1 & 1 & 0 & $\odot$ & $\odot$ & $\odot$ & $\odot$ & L7 \\
\hline$\odot$ & 0 & 0 & $\odot$ & $\odot$ & 0 & $\odot$ & 1 & $\odot$ & 0 & $\odot$ & $\odot$ & $\odot$ & $\odot$ & $\odot$ & $\odot$ & $\odot$ & 0 & 0 & $\odot$ & 1 & -1 & $\odot$ & $\odot$ & 0 & $\odot$ & $\odot$ & $\odot$ & L8 \\
\hline 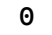 & 0 & 0 & 0 & 0 & 0 & 0 & 0 & 1 & 1 & 0 & 0 & 0 & 0 & 0 & 0 & 0 & 0 & 0 & -1 & 1 & 0 & 1 & 1 & -1 & 0 & 0 & 0 & L9 \\
\hline 0 & 0 & 0 & 0 & 0 & 0 & 0 & 0 & 0 & 0 & 1 & 0 & 0 & 0 & 0 & 0 & 0 & 1 & 0 & 0 & 0 & 0 & -1 & 0 & 0 & 0 & 0 & 0 & L10 \\
\hline 0 & 0 & 0 & $\odot$ & 0 & 0 & 0 & 0 & $\odot$ & 0 & 0 & 1 & 0 & 0 & 0 & 0 & 0 & 0 & 0 & -1 & 1 & 0 & 0 & 0 & 0 & 0 & $\odot$ & $\odot$ & L11 \\
\hline$\odot$ & 0 & 0 & $\odot$ & $\odot$ & 0 & $\odot$ & 0 & $\odot$ & $\odot$ & $\odot$ & $\odot$ & $\odot$ & 1 & 0 & 0 & $\odot$ & -1 & $\odot$ & -1 & 1 & $\odot$ & $\odot$ & -1 & 1 & $\odot$ & $\odot$ & 0 & L12 \\
\hline 0 & 0 & 0 & 0 & 0 & 0 & 0 & $\odot$ & $\odot$ & $\odot$ & 0 & $\odot$ & ○ & $\odot$ & 1 & $\odot$ & $\odot$ & -1 & 0 & -1 & 1 & $\odot$ & 0 & $\odot$ & $\odot$ & $\odot$ & 1 & -1 & L13 \\
\hline$\odot$ & $\odot$ & $\odot$ & $\odot$ & $\odot$ & 0 & $\odot$ & 0 & $\odot$ & $\odot$ & $\odot$ & $\odot$ & $\odot$ & 0 & 0 & 1 & $\odot$ & 1 & $\odot$ & 0 & $\odot$ & $\odot$ & $\odot$ & 0 & $\odot$ & $\odot$ & -1 & 1 & L14 \\
\hline$\odot$ & 0 & 0 & 0 & 0 & 0 & $\odot$ & 0 & 0 & 0 & 0 & 0 & 0 & 0 & 0 & 0 & 1 & 1 & 0 & 0 & -1 & -1 & -1 & 0 & -1 & $\odot$ & -1 & 1 & L15 \\
\hline$\odot$ & 0 & 0 & 0 & 0 & 0 & 0 & 0 & 0 & 0 & 0 & 0 & 0 & 0 & 0 & 0 & 0 & 0 & 1 & -1 & 1 & 0 & 0 & 0 & 0 & 1 & 1 & 0 & L16 \\
\hline$R$ & 2 & 3 & 4 & $5 \mathrm{R}$ & 6 & 7 & 8 & $9 \mathrm{R}$ & 10 & 11 & $12 \mathrm{R}$ & $13 \mathrm{R}$ & 14 & 15 & 16 & 17 & 18 & 19 & 20 & 21 & 22 & 23 & 24 & 25 & $26 \mathrm{R}$ & 27 & 28 & $\square$ \\
\hline
\end{tabular}

None of the above loops is illegal, but they are all linked. Columns corresponding to reversible steps are labeled with the suffix R. To test for illegal linear combinations, columns corresponding to reversible steps are removed, an identity matrix is added, and a second row reduction is performed: 


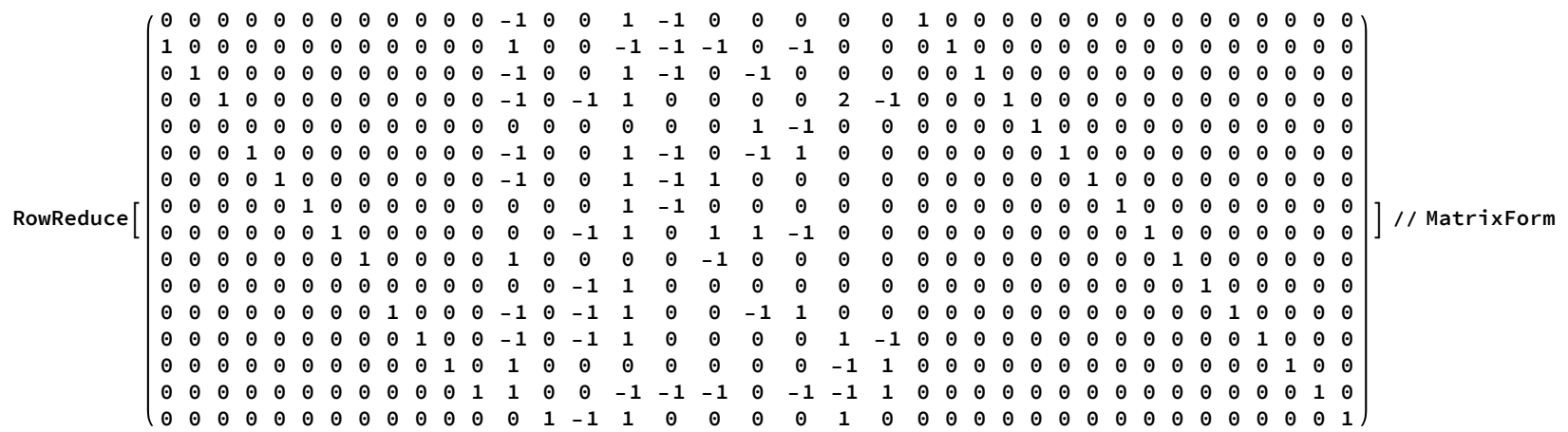

The row reduced matrix is

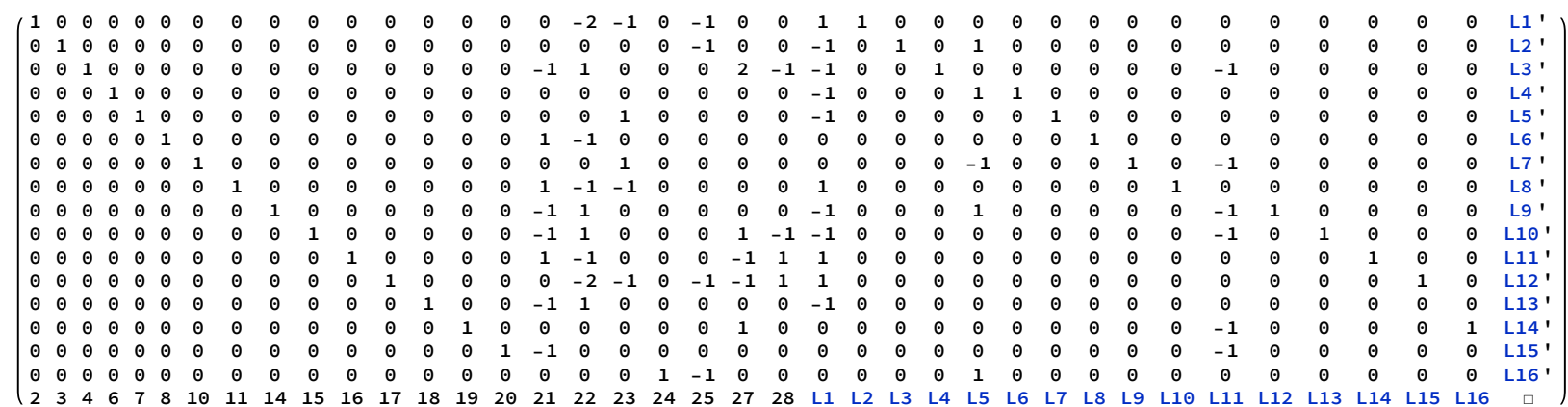

In this matrix the rows are designated as L1' through L16'; the bottom row indicates the irreversible step numbers on the left and the original loop designations of the right. Loops L4', L5', L7', and L14' can be seen to be illegal, since their non-zero coefficients in the left-hand side of the matrix all have the same sign. The right-hand side of the matrix shows the linear combinations forming the loops: L4' $=-\mathrm{L} 1+\mathrm{L} 5+\mathrm{L} 6$; L5' $=-\mathrm{L} 1+\mathrm{L} 7$; L7' $=-\mathrm{L} 5$ + L9 - L11; $\mathrm{L}_{14}{ }^{\prime}=-\mathrm{L} 11+\mathrm{L} 16$. The composition of the illegal loops is then found by combining the relevant loops from the first row reduction. 


\section{Example of an illegal loop identified by Linear Programming}

Further analysis of the periodate/thiosulfate reaction by linear programming reveals an additional illegal loop. The left-hand side of the above matrix is prepared to be transposed as in

$\operatorname{Transpose}\left[\begin{array}{cccccccccccccccccccccc}1 & 0 & 0 & 0 & 0 & 0 & 0 & 0 & 0 & 0 & 0 & 0 & 0 & 0 & 0 & 0 & -2 & -1 & 0 & -1 & 0 & 0 \\ 0 & 1 & 0 & 0 & 0 & 0 & 0 & 0 & 0 & 0 & 0 & 0 & 0 & 0 & 0 & 0 & 0 & 0 & 0 & -1 & 0 & 0 \\ 0 & 0 & 1 & 0 & 0 & 0 & 0 & 0 & 0 & 0 & 0 & 0 & 0 & 0 & 0 & -1 & 1 & 0 & 0 & 0 & 2 & -1 \\ 0 & 0 & 0 & 1 & 0 & 0 & 0 & 0 & 0 & 0 & 0 & 0 & 0 & 0 & 0 & 0 & 0 & 0 & 0 & 0 & 0 & 0 \\ 0 & 0 & 0 & 0 & 1 & 0 & 0 & 0 & 0 & 0 & 0 & 0 & 0 & 0 & 0 & 0 & 0 & 1 & 0 & 0 & 0 & 0 \\ 0 & 0 & 0 & 0 & 0 & 1 & 0 & 0 & 0 & 0 & 0 & 0 & 0 & 0 & 0 & 1 & -1 & 0 & 0 & 0 & 0 & 0 \\ 0 & 0 & 0 & 0 & 0 & 0 & 1 & 0 & 0 & 0 & 0 & 0 & 0 & 0 & 0 & 0 & 0 & 1 & 0 & 0 & 0 & 0 \\ 0 & 0 & 0 & 0 & 0 & 0 & 0 & 1 & 0 & 0 & 0 & 0 & 0 & 0 & 0 & 1 & -1 & -1 & 0 & 0 & 0 & 0 \\ 0 & 0 & 0 & 0 & 0 & 0 & 0 & 0 & 1 & 0 & 0 & 0 & 0 & 0 & 0 & -1 & 1 & 0 & 0 & 0 & 0 & 0 \\ 0 & 0 & 0 & 0 & 0 & 0 & 0 & 0 & 0 & 1 & 0 & 0 & 0 & 0 & 0 & -1 & 1 & 0 & 0 & 0 & 1 & -1 \\ 0 & 0 & 0 & 0 & 0 & 0 & 0 & 0 & 0 & 0 & 1 & 0 & 0 & 0 & 0 & 1 & -1 & 0 & 0 & 0 & -1 & 1 \\ 0 & 0 & 0 & 0 & 0 & 0 & 0 & 0 & 0 & 0 & 0 & 1 & 0 & 0 & 0 & 0 & -2 & -1 & 0 & -1 & -1 & 1 \\ 0 & 0 & 0 & 0 & 0 & 0 & 0 & 0 & 0 & 0 & 0 & 0 & 1 & 0 & 0 & -1 & 1 & 0 & 0 & 0 & 0 & 0 \\ 0 & 0 & 0 & 0 & 0 & 0 & 0 & 0 & 0 & 0 & 0 & 0 & 0 & 1 & 0 & 0 & 0 & 0 & 0 & 0 & 1 & 0 \\ 0 & 0 & 0 & 0 & 0 & 0 & 0 & 0 & 0 & 0 & 0 & 0 & 0 & 0 & 1 & -1 & 0 & 0 & 0 & 0 & 0 & 0 \\ 0 & 0 & 0 & 0 & 0 & 0 & 0 & 0 & 0 & 0 & 0 & 0 & 0 & 0 & 0 & 0 & 0 & 0 & 1 & -1 & 0 & 0\end{array}\right)$ //MatrixForm

After transposing, a row of -1 is added at the bottom to yield

$$
\left(\begin{array}{cccccccccccccccc}
1 & 0 & 0 & 0 & 0 & 0 & 0 & 0 & 0 & 0 & 0 & 0 & 0 & 0 & 0 & 0 \\
0 & 1 & 0 & 0 & 0 & 0 & 0 & 0 & 0 & 0 & 0 & 0 & 0 & 0 & 0 & 0 \\
0 & 0 & 1 & 0 & 0 & 0 & 0 & 0 & 0 & 0 & 0 & 0 & 0 & 0 & 0 & 0 \\
0 & 0 & 0 & 1 & 0 & 0 & 0 & 0 & 0 & 0 & 0 & 0 & 0 & 0 & 0 & 0 \\
0 & 0 & 0 & 0 & 1 & 0 & 0 & 0 & 0 & 0 & 0 & 0 & 0 & 0 & 0 & 0 \\
0 & 0 & 0 & 0 & 0 & 1 & 0 & 0 & 0 & 0 & 0 & 0 & 0 & 0 & 0 & 0 \\
0 & 0 & 0 & 0 & 0 & 0 & 1 & 0 & 0 & 0 & 0 & 0 & 0 & 0 & 0 & 0 \\
0 & 0 & 0 & 0 & 0 & 0 & 0 & 1 & 0 & 0 & 0 & 0 & 0 & 0 & 0 & 0 \\
0 & 0 & 0 & 0 & 0 & 0 & 0 & 0 & 1 & 0 & 0 & 0 & 0 & 0 & 0 & 0 \\
0 & 0 & 0 & 0 & 0 & 0 & 0 & 0 & 0 & 1 & 0 & 0 & 0 & 0 & 0 & 0 \\
0 & 0 & 0 & 0 & 0 & 0 & 0 & 0 & 0 & 0 & 1 & 0 & 0 & 0 & 0 & 0 \\
0 & 0 & 0 & 0 & 0 & 0 & 0 & 0 & 0 & 0 & 0 & 1 & 0 & 0 & 0 & 0 \\
0 & 0 & 0 & 0 & 0 & 0 & 0 & 0 & 0 & 0 & 0 & 0 & 1 & 0 & 0 & 0 \\
0 & 0 & 0 & 0 & 0 & 0 & 0 & 0 & 0 & 0 & 0 & 0 & 0 & 1 & 0 & 0 \\
0 & 0 & 0 & 0 & 0 & 0 & 0 & 0 & 0 & 0 & 0 & 0 & 0 & 0 & 1 & 0 \\
0 & 0 & -1 & 0 & 0 & 1 & 0 & 1 & -1 & -1 & 1 & 0 & -1 & 0 & -1 & 0 \\
-2 & 0 & 1 & 0 & 0 & -1 & 0 & -1 & 1 & 1 & -1 & -2 & 1 & 0 & 0 & 0 \\
-1 & 0 & 0 & 0 & 1 & 0 & 1 & -1 & 0 & 0 & 0 & -1 & 0 & 0 & 0 & 0 \\
0 & 0 & 0 & 0 & 0 & 0 & 0 & 0 & 0 & 0 & 0 & 0 & 0 & 0 & 0 & 1 \\
-1 & -1 & 0 & 0 & 0 & 0 & 0 & 0 & 0 & 0 & 0 & -1 & 0 & 0 & 0 & -1 \\
0 & 0 & 2 & 0 & 0 & 0 & 0 & 0 & 0 & 1 & -1 & -1 & 0 & 1 & 0 & 0 \\
0 & 0 & -1 & 0 & 0 & 0 & 0 & 0 & 0 & -1 & 1 & 1 & 0 & 0 & 0 & 0 \\
-1 & -1 & -1 & -1 & -1 & -1 & -1 & -1 & -1 & -1 & -1 & -1 & -1 & -1 & -1 & -1
\end{array}\right)
$$


Construct the c vector:

$\{-1,-1,-1,-1,-1,-1,-1,-1,-1,-1,-1,-1,-1,-1,-1,-1\}$

Construct the $\mathrm{b}$ vector:

$\{0,0,0,0,0,0,0,0,0,0,0,0,0,0,0,0,0,0,0,0,0,0,-120\}$

Linear programming results in the vector

$\{\odot, \odot, \odot, \odot, 4 \odot, \odot, \odot, 4 \odot, 4 \odot, \odot, \odot, \odot, \odot, \odot, \odot, \odot\}$

The dot product of this vector and the $\mathrm{c}$ vector is -120 , which is proof of the existence of at least one illegal loop.

The vector arising from the LinearProgramming operation indicates the composition of the illegal loop. The non-zero elements of this vector are the coefficients in the linear combination of rows in the matrix of row-reduced irreversible loops forming the illegal loop. Thus, the linear combination of L5' + L8' + L9' contains the irreversible steps of an illegal loop. The complete set of steps in the illegal loop can be determined by recognizing that L5' $=-\mathrm{L} 1+\mathrm{L} 7, \mathrm{~L} 8^{\prime}=\mathrm{L} 1+\mathrm{L} 10$, and $\mathrm{L}^{\prime}=-\mathrm{L} 1+\mathrm{L} 5-\mathrm{L} 11+\mathrm{L} 12$; thus the illegal loop is $-\mathrm{L} 1+\mathrm{L} 5+\mathrm{L} 7+\mathrm{L} 10-\mathrm{L} 11+\mathrm{L} 12$. Combining the steps of these component loops yields the illegal loop: $-1 R+5 R+7+11-12 R$ +14 . The three irreversible steps in this loop all proceed to the right, and thus it is illegal.

\section{References}

(1) Adigun, R. A.; Mhike, M.; Mbiya, W.; Jonnalagadda, S. B.; Simoyi, R. H. OxyhalogenSulfur Chemistry: Kinetics and Mechanism of Oxidation of Chemoprotectant, Sodium 2Mercaptoethanesulfonate, MESNA, by Acidic Bromate and Aqueous Bromine. J. Phys. Chem. A, 2014, 118, 2196-2208.

(2) Orbán, M.; Kurin-Csörgei, Räbai, G.; Epstein, I. R. Mechanistic studies of oscillatory copper(II) catalyzed oxidation reactions of sulfur compounds. Chem. Eng. Sci., 2000, 55, 267-273.

(3) Rauscher, E.; Csekö, G.; Horváth, A. K. On the Complexity of Kinetics and the Mechanism of the Thiosulfate-Periodate Reaction. Inorg. Chem., 2011, 50, 5793-5802. 